\title{
EXPLICIT SEMIANALYTICAL THEORY OF ASTEROID MOTION
}

\author{
I.V. TUPIKOVA, A.A. VAKHIDOV and M. SOFFEL \\ Lohrmann Observatory, Technical University Dresden, Germany
}

\begin{abstract}
A new semianalytical theory of asteroid motion is presented. The theory is developed on the basis of Kaula's expansion of the disturbing function including terms up to the second order with respect to the masses of disturbing bodies. The theory is constructed in explicit form that gives the possibility to study separately the influence of different perturbations in the dynamics of minor planets. The mean-motion resonances with major planets as well as mixed three-body resonances can also be taken into account. For the non-resonant case the formulas obtained can be used for deriving the second transformation to calculate the proper elements of an asteroid orbit in closed form with respect to inclinations and eccentricities.
\end{abstract}

Key words: minor planets, resonances, Hamiltonian systems, Lie series

\section{Introduction}

In order to deal with the long-time behaviour of asteroids, one of the modern fast and accurate integration schemes for the equations of motion in Cartesian coordinates might be employed. In the case when we are interested in some special perturbations, we can calculate the amplitudes at certain frequencies with the aid of fast Fourier transform. But we cannot tell for sure, which effect or interplay of effects has really caused this calculated value of amplitudes (see, for example, the discussion concerning Veritas (Milani et al., 1997; Nesvorny and Morbidelli, 1999). For some problems it might be more efficient or more informative to use a semianalytical approach that gives the averaged equations of motion in explicit form. The averaging procedure is connected with the elimination of short-periodic terms from the initial Hamiltonian describing the motion of asteroids under the influence of selected planets. This procedure can be realized, for example, by means of Lie-series methods ( Hori, 1966; Deprit, 1969). As a result, we obtain the averaged Hamiltonian which contains only secular, long-periodic and resonant terms. The number of these terms (at least in the first two succesive approximations with respect to the small parameter of the problem) is substantially less than the number of short-periodic terms which are eliminated from the Hamiltonian. Finally, we can integrate the system of the averaged differential equations of motion numerically.

The main advantages of semianalytical methods in comparison with the direct numerical integration of equations of motion are connected with the possibilities:

- to eliminate the non-essential short-periodic perturbations from the Hamiltonian (and this is possible up to any desired order in the small parameter of the problem);

- to investigate the "pure" dynamical picture defined only by secular, longperiodic and resonant perturbations;

— to increase substantially the step-size of numerical integration. 
Here, we present a new semianalytical theory which can be used for numerical integration of averaged equations of motion as well as for the construction of the second transformation for the derivation of proper elements of asteroid orbits in the non-resonant case. The theory considers the perturbations of the second order explicitly that allows to select certain resonant harmonics (e.g. certain mixed resonances, see Nesvorny and Morbidelli, 1999) and to reveal a minimal set of perturbations causing certain characteristics in the observed orbital dynamics. Since we have employed Kaula's expansion of the disturbing function, the solution is obtained in closed form w.r.t. inclinations (via inclination functions) and eccentricites (via Hansen coefficients). It is to be understood that the theory can be used only for the case of reasonable convergency with respect to the parameter $\alpha$ (ratio of semi-major axes).

\section{Averaging procedure}

For the construction of perturbation theory of the second order we use the averaging procedure based on the Deprit modification of the Lie-series method (Deprit, 1969); in this procedure the short-periodic perturbations supposedly non-essential are eliminated.

Let us consider the Hamiltonian of the problem in the form

$$
F=F_{0}+F_{1},
$$

with the unperturbed part

$$
\begin{aligned}
F_{0} & =\frac{\mu^{2}}{2 L^{2}}-K, \\
\mu & =G_{0} \mathcal{M}_{\text {Sun }},
\end{aligned}
$$

( $G_{0}$ is the Newton's gravitational constant, $\mathcal{M}$ stands for mass) and with the disturbing function which can be expressed in the classical form (Kaula, 1966; Yuasa, 1973)

$$
\begin{array}{r}
F_{1}=\sum_{B} \sum_{i=\left(i_{1}, \ldots, i_{6}\right)} f_{i}^{B}\left(L, G, H, L_{B}, G_{B}, H_{B}\right) \cos \phi_{i}^{B}, \\
\phi_{i}^{B}=i_{1} l+i_{2} l_{B}+i_{3} g+i_{4} g_{B}+i_{5} h+i_{6} h_{B},
\end{array}
$$

in the extended phase space of variables $(L, G, H, K, l, g, h, t)$. Here $\mathrm{K}$ is conjugate to the time variable $t$ and $L, G, H, l, g, h$ are the usual Delaunay elements. The index $B$ refers to the perturbing body. The motions of the perturbing bodies are supposed to be known functions of $t$; then the disturbing function (1) depends explicitly upon the time only via the elements $L_{B}, G_{B}, H_{B}, l_{B}, g_{B}, h_{B}$.

We assume that $F_{1}$ is of the order of the small parameter $\mathcal{M}_{B} / \mathcal{M}_{\text {Sun }}$ which allows to construct a perturbation theory by means of successive approximations. 
According to Deprit's method in the first approximation we should solve the differential equation

$$
\left\{F_{0}, W_{1}\right\}+F_{1}=F_{1}^{*}
$$

(where $\left\{F_{0}, W_{1}\right\}$ are the Poisson brackets in the extended phase space) in order to derive the generating function $W_{1}$ of the corresponding canonical transformation.

We would like to point out that in the formulae for the determination of the new Hamiltonian and the generating function in all the components the new (mean) elements should be substituted instead of the old (osculating) ones; below we will omit that for simplicity.

We put

$$
F_{1}^{*}=\left[F_{1}\right]_{\text {sec }}
$$

as the part of the initial Hamiltonian which contains only secular, long-periodic and resonant terms (in the case of the commensurability between the mean motion of the asteroid $\bar{n}$ and that of the perturbing body $\bar{n}_{B}$ defined by $\bar{n}: \bar{n}_{B} \approx \gamma: \gamma_{B}$, where $\gamma, \gamma_{B}$ are some integers). Then the Hamilton-Jacobi equation for the generating function $W_{1}$ has the following form:

$$
-\bar{n} \frac{\partial W_{1}}{\partial l}-\frac{\partial W_{1}}{\partial t}+\left[F_{1}\right]_{\text {short-per }}=0 .
$$

Here $\left[F_{1}\right]_{\text {short-per }}$ is the part of initial Hamiltonian (1) containing only shortperiodic terms.

Putting $l_{B}=\bar{n}_{B} t+l_{B}^{0}$ (here $l_{B}^{0}$ is a constant), we find:

$$
W_{1}=\sum_{B} \sum_{i=\left(i_{1}, \ldots, i_{6}\right)} \Delta_{i}^{B} f_{i}^{B} \sin \phi_{i}^{B}
$$

To simplify the further computations we introduce here

$$
\Delta_{i}^{B}= \begin{cases}0, & \text { for secular, long-periodic } \\ & \text { and resonant (with body "B") terms } \\ 1 /\left(i_{1} \bar{n}+i_{2} \bar{n}_{B}\right), & \text { otherwise. }\end{cases}
$$

One can show that neglecting the explicit dependence of $g_{B}$ and $h_{B}$ upon time when solving equation (2) introduces an error of the third order.

In the second order we have the following Hamilton-Jacobi equation

$$
\left\{F_{0}, W_{2}\right\}+\frac{1}{2}\left\{F_{1}+F_{1}^{*}, W_{1}\right\}=F_{2}^{*}
$$

for the generating function $W_{2}$. In the same way, we define the corresponding part of the second order Hamiltonian which contains only secular, long-periodic and resonant terms as

$$
F_{2}^{*}=\frac{1}{2}\left\{F_{1}+F_{1}^{*}, W_{1}\right\}_{\mathrm{sec}}
$$


to find $W_{2}$ from

$$
-\bar{n} \frac{\partial W_{2}}{\partial l}-\frac{\partial W_{2}}{\partial t}+\frac{1}{2}\left\{F_{1}+F_{1}^{*}, W_{1}\right\}_{\text {short-per }}=0 .
$$

Note that $F_{2}^{*}$ and $W_{2}$ contain the mixed terms arising from the interaction of two perturbing bodies with the asteroid.

For two bodies (e.g., Jupiter $(J)$ and Saturn $(S)$ ) we have

$$
\begin{gathered}
F_{1}=F_{1}^{J}+F_{1}^{S}, \quad F_{1}^{*}=F_{1}^{J *}+F_{1}^{S *}, \\
W_{1}=W_{1}^{J}+W_{1}^{S} .
\end{gathered}
$$

Then the second Poisson bracket on the 1.h.s. of (4) is a sum of four terms:

$$
\begin{aligned}
& \frac{1}{2}\left\{F_{1}^{J}+F_{1}^{J *}+F_{1}^{S}+F_{1}^{S *}, W_{1}^{J}+W_{1}^{S}\right\}= \\
& =\frac{1}{2}\left\{F_{1}^{J}+F_{1}^{J *}, W_{1}^{J}\right\}+\frac{1}{2}\left\{F_{1}^{S}+F_{1}^{S *}, W_{1}^{S}\right\}+ \\
& +\frac{1}{2}\left\{F_{1}^{J}+F_{1}^{J *}, W_{1}^{S}\right\}+\frac{1}{2}\left\{F_{1}^{S}+F_{1}^{S *}, W_{1}^{J}\right\} .
\end{aligned}
$$

The first two terms describe the direct perturbations of the second order from the perturbing bodies (Jupiter or Saturn, respectively).

With $F_{1}^{B}+F_{1}^{B *}$ written as

$$
F_{1}^{B}+F_{1}^{B *}=\sum_{j=\left(j_{1}, \ldots, j_{6}\right)} \delta_{j}^{B} f_{j}^{B} \cos \phi_{j}^{B}
$$

where

$$
\delta_{j}^{B}= \begin{cases}2, & \text { for secular, long-periodic and resonant (with body "B") terms, } \\ 1, & \text { otherwise, }\end{cases}
$$

we find

$$
\begin{aligned}
& \frac{1}{2}\left\{F_{1}^{B}+F_{1}^{B *}, W_{1}^{B}\right\}= \\
& =\frac{1}{4} \sum_{i} \sum_{j} \sum_{k=-1,+1} f_{j}^{B}\left[\left(\frac{\partial f_{i}^{B}}{\partial L} j_{1}+\frac{\partial f_{i}^{B}}{\partial G} j_{3}+\frac{\partial f_{i}^{B}}{\partial H} j_{5}\right)\left(\Delta_{j}^{B} \delta_{i}^{B}+k \Delta_{i}^{B} \delta_{j}^{B}\right)+\right. \\
& \left.+3 f_{i}^{B} \frac{\bar{n}}{L} k\left(\Delta_{i}^{B}\right)^{2} \delta_{j}^{B} i_{1} j_{1}\right] \cos \left(\phi_{i}^{B}-k \phi_{j}^{B}\right) .
\end{aligned}
$$

Two last Poisson brackets in formula (5) represent the mixed perturbations:

$$
\begin{aligned}
& \frac{1}{2}\left\{F_{1}^{J}+F_{1}^{J *}, W_{1}^{S}\right\}+\frac{1}{2}\left\{F_{1}^{S}+F_{1}^{S *}, W_{1}^{J}\right\}= \\
& =\frac{1}{4} \sum_{i} \sum_{j} \sum_{k=-1,+1}\left[\left[f_{j}^{S}\left(\frac{\partial f_{i}^{J}}{\partial L} j_{1}+\frac{\partial f_{i}^{J}}{\partial G} j_{3}+\frac{\partial f_{i}^{J}}{\partial H} j_{5}\right)\right.\right.
\end{aligned}
$$




$$
\begin{aligned}
& \left.+k f_{i}^{J}\left(\frac{\partial f_{j}^{S}}{\partial L} i_{1}+\frac{\partial f_{j}^{S}}{\partial G} i_{3}+\frac{\partial f_{j}^{S}}{\partial H} i_{5}\right)\right]\left(\Delta_{j}^{S} \delta_{i}^{J}+k \Delta_{i}^{J} \delta_{j}^{S}\right)+ \\
& \left.+3 f_{i}^{J} f_{j}^{S} \frac{\bar{n}}{L} k i_{1} j_{1}\left(\left(\Delta_{i}^{J}\right)^{2} \delta_{j}^{S}+\left(\Delta_{j}^{S}\right)^{2} \delta_{i}^{J}\right)\right] \cos \left(\phi_{i}^{J}-k \phi_{j}^{S}\right) .
\end{aligned}
$$

Let us write

$$
F_{2}^{*}=F_{2}^{J *}+F_{2}^{S *}+F_{2}^{J S *},
$$

then $F_{2}^{B *}$ is given by

$$
F_{2}^{B *}=\frac{1}{2}\left\{F_{1}^{B}+F_{1}^{B *}, W_{1}^{B}\right\}_{\mathrm{sec}},
$$

that is, as the part of $(6)$ which contains secular and long-periodic terms determined by the following conditions:

$$
i_{1}-k j_{1}=i_{2}-k j_{2}=0
$$

and in the resonant case $\bar{n}: \bar{n}_{B} \approx \gamma: \gamma_{B}$ also the resonant terms satisfying the equation:

$$
\frac{i_{1}-k j_{1}}{i_{2}-k j_{2}}=-\frac{\gamma_{B}}{\gamma}
$$

The function $F_{2}^{J S *}$ is determined by

$$
F_{2}^{J S *}=\frac{1}{2}\left\{F_{1}^{J}+F_{1}^{J *}, W_{1}^{S}\right\}_{\mathrm{sec}}+\frac{1}{2}\left\{F_{1}^{S}+F_{1}^{S *}, W_{1}^{J}\right\}_{\mathrm{sec}},
$$

that is, as the part of (7) containing only secular, long-periodic and resonant terms. Notice, that the trigonometric arguments in (7)

$$
\begin{gathered}
\phi_{i}^{J}-k \phi_{j}^{S}=\left(i_{1}-k j_{1}\right) l+i_{2} l_{J}-k j_{2} l_{S}+\left(i_{3}-k j_{3}\right) g+\left(i_{5}-k j_{5}\right) h+ \\
+i_{4} g_{J}+i_{6} h_{J}-k j_{4} g_{S}-k j_{6} h_{S}
\end{gathered}
$$

give the resonant components not only in the case of mean motion commensurabilities but also for mixed resonances.

Similarly, let us write $W_{2}$ as

$$
W_{2}=W_{2}^{J}+W_{2}^{S}+W_{2}^{J S}
$$

then $W_{2}^{B} \quad(B=J, S)$ is given by

$$
\begin{aligned}
& W_{2}^{B}=\frac{1}{4} \sum_{i} \sum_{j} \sum_{k=-1,+1} \Delta_{i j}^{B} f_{j}^{B} \times \\
& \times\left[\left(\frac{\partial f_{i}^{B}}{\partial L} j_{1}+\frac{\partial f_{i}^{B}}{\partial G} j_{3}+\frac{\partial f_{i}^{B}}{\partial H} j_{5}\right)\left(\Delta_{j}^{B} \delta_{i}^{B}+k \Delta_{i}^{B} \delta_{j}^{B}\right)+\right. \\
& \left.+3 f_{i}^{B} \frac{\bar{n}}{L} k \Delta_{i}^{B} \delta_{j}^{B} i_{1} j_{1}\right] \sin \left(\phi_{i}^{B}-k \phi_{j}^{B}\right)
\end{aligned}
$$


with

$$
\Delta_{i j}^{B}= \begin{cases}0, & \text { for secular, long-periodic } \\ 1 /\left(\left(i_{1}-k j_{1}\right) \bar{n}+\left(i_{2}-k j_{2}\right) \bar{n}_{B}\right), & \text { and resonant with "B" terms, } \\ \text { otherwise. }\end{cases}
$$

Finally,

$$
\begin{aligned}
& W_{2}^{J S}=\frac{1}{4} \sum_{i} \sum_{j} \sum_{k=-1,+1} \Delta_{i j}^{J S}\left[\left[f_{j}^{S}\left(\frac{\partial f_{i}^{J}}{\partial L} j_{1}+\frac{\partial f_{i}^{J}}{\partial G} j_{3}+\frac{\partial f_{i}^{J}}{\partial H} j_{5}\right)+\right.\right. \\
& \left.+k f_{i}^{J}\left(\frac{\partial f_{j}^{S}}{\partial L} i_{1}+\frac{\partial f_{j}^{S}}{\partial G} i_{3}+\frac{\partial f_{j}^{S}}{\partial H} i_{5}\right)\right]\left(\Delta_{j}^{S} \delta_{i}^{J}+k \Delta_{i}^{J} \delta_{j}^{S}\right)+ \\
& \left.+3 f_{i}^{J} f_{j}^{S} \frac{\bar{n}}{L} k i_{1} j_{1}\left(\left(\Delta_{i}^{J}\right)^{2} \delta_{j}^{S}+\left(\Delta_{j}^{S}\right)^{2} \delta_{i}^{J}\right)\right] \sin \left(\phi_{i}^{J}-k \phi_{j}^{S}\right),
\end{aligned}
$$

where

$$
\Delta_{i j}^{J S}= \begin{cases}0, & \text { for secular, long-periodic and } \\ & \text { resonant terms, } \\ 1 /\left(\left(i_{1}-k j_{1}\right) \bar{n}+i_{2} \bar{n}_{J}-k j_{2} \bar{n}_{S}\right), & \text { otherwise. }\end{cases}
$$

\section{Application to Kaula's Expansion of the Disturbing Function}

For asteroids moving relatively far from the perturbing bodies we will employ Kaula's expansion of the disturbing function (Kaula, 1966; Fominov, 1980) for each perturbing body "B" $\left(\alpha_{B}=a / a_{B}\right)$ :

$$
\begin{aligned}
& F_{1}^{B}=\frac{\mu_{B}}{a_{B}} \sum_{n=2}^{\infty} \sum_{m=0}^{n} \sum_{p=0}^{n} \sum_{h=0}^{n} \sum_{q=-\infty}^{\infty} \sum_{j=-\infty}^{\infty} \frac{\alpha_{B}^{n}}{2 n+1} \times \\
& \times \bar{F}_{n m p}(I) \bar{F}_{n m h}\left(I_{B}\right) H_{n p q}(e) G_{n h j}\left(e_{B}\right) \cos \Phi_{B},
\end{aligned}
$$

where

$$
\begin{aligned}
& \Phi_{B}=(n-2 p+q) M-(n-2 h+j) M_{B}+ \\
& +(n-2 p) \omega-(n-2 h) \omega_{B}+m\left(\Omega-\Omega_{B}\right),
\end{aligned}
$$

and

$$
H_{n p q}(e)=X_{n-2 p+q}^{n, n-2 p}(e), \quad G_{n h j}\left(e_{B}\right)=X_{n-2 h+j}^{-n-1, n-2 h}\left(e_{B}\right) .
$$

Here, $a, e, I, \Omega, \omega, M$ and $a_{B}, e_{B}, I_{B}, \Omega_{B}, \omega_{B}, M_{B}$ are Keplerian elements of the asteroid and disturbing body, respectively; $\bar{F}_{n m p}(I)$ are normalized Kaula inclination functions, $X_{q}^{n, p}(e)$ are the classical Hansen coefficients and $\mu_{B}$ is the gravitational constant of the perturbing body: $\mu_{B}=G_{0} \mathcal{M}_{\mathcal{B}} / \mathcal{M}_{\text {Sun }}$. 
With the aid of the averaging procedure described above we can construct the averaged Hamiltonian up to the second order

$$
\begin{gathered}
F^{*}=F_{0}^{*}+F_{1}^{*}+F_{2}^{*}, \\
F_{1}^{*}=F_{1}^{J *}+F_{1}^{S *}, \quad F_{2}^{*}=F_{2}^{J *}+F_{2}^{S *}+F_{2}^{J S *}
\end{gathered}
$$

and the generating function

$$
\begin{gathered}
W=W_{1}+W_{2}, \\
W_{1}=W_{1}^{J}+W_{1}^{S}, \quad W_{2}=W_{2}^{J}+W_{2}^{S}+W_{2}^{J S} .
\end{gathered}
$$

The part of the Hamiltonian of the first order containing only secular and longperiodic terms reads

$$
\begin{aligned}
& F_{1}^{B *}=\frac{\mu_{B}}{a_{B}} \sum_{n=2}^{\infty} \sum_{m=0}^{n} \sum_{p=0}^{n} \sum_{h=0}^{n} \frac{\alpha_{B}{ }^{n}}{2 n+1} \bar{F}_{n m p}(I) \bar{F}_{n m h}\left(I_{B}\right) X_{0}^{n, n-2 p}(e) \times \\
& \times X_{0}^{-n-1, n-2 h}\left(e_{B}\right) \cos \left[(n-2 p) \omega-(n-2 h) \omega_{B}+m\left(\Omega-\Omega_{B}\right)\right] .
\end{aligned}
$$

Resonant terms appear in development (12) in the case $\bar{n}: \bar{n}_{B} \approx \gamma: \gamma_{B}$, if

$$
\frac{n-2 p+q}{n-2 h+j}=\frac{\gamma_{B}}{\gamma}
$$

In this case the corresponding resonant terms have to be included in $F_{1}^{B *}$. Note, that in (12) there are no summations with respect to indices $q$ and $j$ which reduces the problem of convergency of the series with respect to Hansen coefficients.

According to (3), the generating function $W_{1}^{B}$ of the first order is

$$
\begin{gathered}
W_{1}^{B}=\frac{\mu_{B}}{a_{B}} \sum_{n=2}^{\infty} \sum_{m=0}^{n} \sum_{p=0}^{n} \sum_{h=0}^{n} \sum_{q=-\infty}^{\infty} \sum_{j=-\infty}^{\infty} \alpha_{B}^{n} \times \\
\times \Delta_{n p q h j}^{B} \frac{1}{(2 n+1)} \bar{F}_{n m p}(I) \bar{F}_{n m h}\left(I_{B}\right) H_{n p q}(e) G_{n h j}\left(e_{B}\right) \sin \Phi^{B},
\end{gathered}
$$

where

$$
\Delta_{n p q h j}^{B}= \begin{cases}0, & \text { for secular, long-periodic } \\ 1 /\left((n-2 p+q) \bar{n}-(n-2 h+j) \bar{n}_{B}\right), & \text { and resonant (with B) terms, } \\ & \text { otherwise. }\end{cases}
$$

Using the expressions for the partials $(s=\sin I / 2)$

$$
\frac{\partial}{\partial L}=2 \sqrt{\frac{a}{\mu}} \frac{\partial}{\partial a}+\frac{1-e^{2}}{e L} \frac{\partial}{\partial e},
$$




$$
\begin{aligned}
& \frac{\partial}{\partial G}=\frac{1}{L \sqrt{1-e^{2}}}\left(-\frac{1-e^{2}}{e} \frac{\partial}{\partial e}+\frac{1-2 s^{2}}{4 s} \frac{\partial}{\partial s}\right), \\
& \frac{\partial}{\partial H}=-\frac{1}{L \sqrt{1-e^{2}}} \frac{1}{4 s} \frac{\partial}{\partial s}, \\
& \frac{\partial \bar{n}}{\partial L}=-\frac{3 \bar{n}}{L},
\end{aligned}
$$

from (6) and (8) we get $F_{2}^{B *}$ in the form

$$
\begin{aligned}
& F_{2}^{B *}=\frac{1}{4 L} \frac{\mu_{B}^{2}}{a_{B}^{2}} \sum_{n m p h q j} \sum_{n^{\prime} m^{\prime} p^{\prime} h^{\prime} q^{\prime} j^{\prime}} \sum_{k=-1,+1}{ }^{*} \alpha_{B}^{n+n^{\prime}} \frac{1}{2 n+1} \frac{1}{2 n^{\prime}+1} \times \\
& \times \bar{F}_{n m h}\left(I_{B}\right) \bar{F}_{n^{\prime} m^{\prime} h^{\prime}}\left(I_{B}\right) G_{n h j}\left(e_{B}\right) G_{n^{\prime} h^{\prime} j^{\prime}}\left(e_{B}\right) \bar{F}_{n^{\prime} m^{\prime} p^{\prime}}(I) H_{n^{\prime} p^{\prime} q^{\prime}}(e) \times \\
& \times\left[\bar{F}_{n m p}(I) H_{n p q}(e) \Delta_{2}^{B}+\right. \\
& \left.+\left(\bar{F}_{n m p}(I) H_{n p q}^{1}(e)+\frac{1}{\sqrt{1-e^{2}}} H_{n p q}(e) \bar{F}_{n m p}^{1}(I)\right) \Delta_{1}^{B}\right] \times \\
& \times \cos \left[\left(n-2 p+q-k\left(n^{\prime}-2 p^{\prime}+q^{\prime}\right)\right) M+\left(n-2 p-k\left(n^{\prime}-2 p^{\prime}\right)\right) \omega-\right. \\
& -\left(n-2 h+j-k\left(n^{\prime}-2 h^{\prime}+j^{\prime}\right)\right) M_{B}-\left(n-2 h-k\left(n^{\prime}-2 h^{\prime}\right)\right) \omega_{B}+ \\
& \left.+\left(m-k m^{\prime}\right)\left(\Omega-\Omega_{B}\right)\right] .
\end{aligned}
$$

Here,

$$
\begin{aligned}
& \sum_{n m p h q j}=\sum_{n=2}^{\infty} \sum_{m=0}^{n} \sum_{p=0}^{n} \sum_{h=0}^{n} \sum_{q=-\infty}^{\infty} \sum_{j=-\infty}^{\infty}, \\
& \sum_{n^{\prime} m^{\prime} p^{\prime} h^{\prime} q^{\prime} j^{\prime}}=\sum_{n^{\prime}=2}^{\infty} \sum_{m^{\prime}=0}^{n^{\prime}} \sum_{p^{\prime}=0}^{n^{\prime}} \sum_{h^{\prime}=0}^{n^{\prime}} \sum_{q^{\prime}=-\infty}^{\infty} \sum_{j^{\prime}=-\infty}^{\infty}, \\
& H_{n p q}^{1}=\left[\left(n^{\prime}-2 p^{\prime}+q^{\prime}\right)\left(1-e^{2}\right)-\left(n^{\prime}-2 p^{\prime}\right) \sqrt{\left.1-e^{2}\right]} \frac{1}{e} \frac{\partial H_{n p q}}{\partial e}\right. \\
& \bar{F}_{n m p}^{1}=\frac{1}{4}\left[\left(n^{\prime}-2 p^{\prime}\right)\left(1-2 s^{2}\right)-m^{\prime}\right] \frac{1}{s} \frac{\partial \bar{F}_{n m p}}{\partial s}, \\
& \Delta_{1}^{B}=\Delta_{n^{\prime} p^{\prime} q^{\prime} h^{\prime} j^{\prime}}^{B} \delta_{n p q h j}^{B}+k \Delta_{n p q h j}^{B} \delta_{n^{\prime} p^{\prime} q^{\prime} h^{\prime} j^{\prime}}, \\
& \Delta_{2}^{B}=2 n\left(n^{\prime}-2 p^{\prime}+q^{\prime}\right) \Delta_{1}^{B}+ \\
& +3 k \bar{n}(n-2 p+q)\left(n^{\prime}-2 p^{\prime}+q^{\prime}\right)\left(\Delta_{n p q h j}^{B}\right)^{2} \delta_{n^{\prime} p^{\prime} q^{\prime} h^{\prime} j^{\prime}}^{B},
\end{aligned}
$$




$$
\delta_{n p q h j}^{B}= \begin{cases}2, \quad & \text { if } n-2 p+q=n-2 h+j=0 \\ & \text { or }(n-2 p+q):(n-2 h+j)=\gamma_{B}: \gamma \\ & \text { in the resonant case, } \\ & \text { otherwise, }\end{cases}
$$

$\Delta_{n^{\prime} p^{\prime} q^{\prime} h^{\prime} j^{\prime}}^{B}$ and $\delta_{n^{\prime} p^{\prime} q^{\prime} h^{\prime} j^{\prime}}^{B}$ are defined by the formulae for $\Delta_{n p q h j}^{B}$ and $\delta_{n p q h j}^{B}$ by putting primes at the indices, and $*$ at the summation symbol means that one should take only the terms with the indices satisfying the conditions: $n-2 p+q-$ $k\left(n^{\prime}-2 p^{\prime}+q^{\prime}\right)=n-2 h+j-k\left(n^{\prime}-2 h^{\prime}+j^{\prime}\right)=0$ or

$$
\frac{n-2 p+q-k\left(n^{\prime}-2 p^{\prime}+q^{\prime}\right)}{n-2 h+j-k\left(n^{\prime}-2 h^{\prime}+j^{\prime}\right)}=\frac{\gamma_{B}}{\gamma} \text {. }
$$

Finally, by means of (7) and (9) we get

$$
\begin{aligned}
& F_{2}^{J S *}=\frac{1}{4 L} \frac{\mu_{J}}{a_{J}} \frac{\mu_{S}}{a_{S}} \sum_{n m p h q j} \sum_{n^{\prime} m^{\prime} p^{\prime} h^{\prime} q^{\prime} j^{\prime}} \sum_{k=-1,+1}{ }^{* *} \alpha_{J}^{n} \alpha_{S}^{n^{\prime}} \times \\
& \times \frac{1}{(2 n+1)} \frac{1}{\left(2 n^{\prime}+1\right)} \bar{F}_{n m h}\left(I_{J}\right) \bar{F}_{n^{\prime} m^{\prime} h^{\prime}}\left(I_{S}\right) G_{n h j}(e J) G_{n^{\prime} h^{\prime} j^{\prime}}\left(e_{S}\right) \times \\
& \times\left[\bar{F}_{n^{\prime} m^{\prime} p^{\prime}}(I) \bar{F}_{n m p}(I) H_{n p q}(e) H_{n^{\prime} p^{\prime} q^{\prime}}(e) \Delta_{2}^{J, S}+\right. \\
& +\bar{F}_{n m p}(I) \bar{F}_{n^{\prime} m^{\prime} p^{\prime}}(I)\left[H_{n p q}^{1}(e) H_{n^{\prime} p^{\prime} q^{\prime}}(e)+k H_{n p q}(e)_{n^{\prime} p^{\prime} q^{\prime}}^{1}(e)\right] \Delta_{1}^{J, S}+ \\
& +\frac{1}{\sqrt{1-e^{2}}} H_{n p q}(e) H_{n^{\prime} p^{\prime} q^{\prime}}(e) \times \\
& \left.\left.\times\left[\bar{F}_{n m p}^{1}(I)\right) \bar{F}_{n^{\prime} m^{\prime} p^{\prime}}(I)+k \bar{F}_{n m p}(I) \bar{F}_{n^{\prime} m^{\prime} p^{\prime}}^{1}(I)\right] \Delta_{1}^{S, J}\right] \times \\
& \times \cos \left[\left(n-2 p+q-k\left(n^{\prime}-2 p^{\prime}+q^{\prime}\right)\right) M^{\prime}+\left(n-2 p-k\left(n^{\prime}-2 p^{\prime}\right)\right) \omega-\right. \\
& -(n-2 h+j) M_{J}+k\left(n^{\prime}-2 h^{\prime}+j^{\prime}\right) M_{S}-(n-2 h) \omega_{J}+k\left(n^{\prime}-2 h^{\prime}\right) \omega_{S}+ \\
& \left.\left.+\left(m-k m^{\prime}\right) \Omega-m \Omega_{J}+k m^{\prime} \Omega_{S}\right)\right]
\end{aligned}
$$

where

$$
\begin{aligned}
& \Delta_{1}^{J, S}=\Delta_{n^{\prime} p^{\prime} q^{\prime} h^{\prime} j^{\prime}}^{S} \delta_{n p q h j}^{J}+k \Delta_{n p q h j}^{J} \delta_{n^{\prime} p^{\prime} q^{\prime} h^{\prime} j^{\prime}}^{S} \\
& \quad \Delta_{2}^{J, S}=\left(2 n\left(n^{\prime}-2 p^{\prime}+q^{\prime}\right)+2 k n^{\prime}(n-2 p+q)\right) \Delta_{1}^{J, S}+ \\
& \quad+3 k \bar{n}(n-2 p+q)\left(n^{\prime}-2 p^{\prime}+q^{\prime}\right)\left[\left(\Delta_{n p q h j}^{J}\right)^{2} \delta_{n^{\prime} p^{\prime} q^{\prime} h^{\prime} j^{\prime}}^{S}+\left(\Delta_{n^{\prime} p^{\prime} q^{\prime} h^{\prime} j^{\prime}}^{S}\right)^{2} \delta_{n p q h j}^{J}\right], \\
& H_{n^{\prime} p^{\prime} q^{\prime}}^{1}=\left[(n-2 p+q)\left(1-e^{2}\right)-(n-2 p) \sqrt{\left.1-e^{2}\right]} \frac{1}{e} \frac{\partial H_{n^{\prime} p^{\prime} q^{\prime}}}{\partial e}\right. \\
& \bar{F}_{n^{\prime} m^{\prime} p^{\prime}}^{1}=\frac{1}{4}\left[(n-2 p)\left(1-2 s^{2}\right)-m\right] \frac{1}{s} \frac{\partial \bar{F}_{n^{\prime} m^{\prime} p^{\prime}}}{\partial s} .
\end{aligned}
$$


Here $* *$ by sums means that one should take only the terms with the indices satisfying the conditions $n-2 p+q-k\left(n^{\prime}-2 p^{\prime}+q^{\prime}\right)=n-2 h+j=n^{\prime}-2 h^{\prime}+j^{\prime}=$ 0 or satisfying the conditions of a mean-motion or three-body resonance. The components of the generating functions $W_{2}^{B}$ can be easily deduced from formula (10) and the function $W_{2}^{J S}$ - from formula (11).

These expressions give the explicit second-order solution for the secular, longperiodic and resonant (also mixed-resonant) perturbations.

Presently, we are in the process to implement the perturbations in the motion of disturbing bodies and then to apply this formalism to the problem of mixedresonances and other perturbations of the second order in the motion of asteroids. We are also planning to develop explicitly the second transformation to exclude the remaining angular variables and to get the analytical solution in the non-resonant case.

\section{Acknowledgements}

The authors are very grateful to Dr. S.Klioner and Dr. M.Šidlichovsky for some useful discussions concerning our work. We would like to express also our gratitude to Prof. R.Dvorak and Dr. E.Pilat-Lohinger for the help in our research and many useful suggestions. We thank also Dr. C.Ron for his attention to our work. A.V. thanks also the German Academician Exchange Service (DAAD) for financial support of this research.

\section{References}

Milani, A., Nobili, A., Knezevic, Z.: 1997, Icarus, 125, 13.

Nesvorny, D., Morbidelli A.: 1987, Astron. J, in press.

Hori, G.I.: 1966, Publ. Astron. Soc. Japan, 18, 287.

Deprit, A.: 1969, Celest. Mech., 1, 12.

Kaula, W.M.: 1966, Theory of Satellite Geodesy, Blaisdell Publ. Co., Mass.

Yuasa, M.: 1973, Publ. Astron. Soc. Japan, 25, 39.9.

Fominov A.M.: 1980, Bull. ITA, 10, 621. 